\title{
Calculation of the Rydberg Energy Levels for Francium Atom
}

\author{
Huang Shizhong and Sun Qiufeng \\ College of Physics and Electrical Information, Anhui Normal University, Wuhu 241000, China \\ Correspondence should be addressed to Huang Shizhong, huangsz@mail.ahnu.edu.cn
}

Received 18 September 2010; Revised 9 November 2010; Accepted 11 November 2010

Academic Editor: N. Bigelow

Copyright () 2010 H. Shizhong and S. Qiufeng. This is an open access article distributed under the Creative Commons Attribution License, which permits unrestricted use, distribution, and reproduction in any medium, provided the original work is properly cited.

Based on the weakest bound electron potential model theory, the Rydberg energy levels and quantum defects of $n s^{2} S_{1 / 2}(n=8-50)$, $n d^{2} \mathrm{D}_{3 / 2}(n=6-50)$, and $n d^{2} \mathrm{D}_{5 / 2}(n=6-50)$ spectrum series for francium atom are calculated. The calculated results are in excellent agreement with the 74 known experimentally measured levels (the absolute difference is less than $0.03 \mathrm{~cm}^{-1}$ ) and 58 energy levels for highly excited states are predicted.

\section{Introduction}

Much work [1-19] has been carried out to investigate the energy structure in excited states of francium atom, the heaviest alkali metal atom. The experimental investigations of the francium spectrum were pioneered by Liberman et al. in 1978 [1]. Later on, the energy levels of $7 \mathrm{p}^{2} \mathrm{P}_{1 / 2,3 / 2}^{0}$ and $8 \mathrm{p}^{2} \mathrm{P}_{1 / 2,3 / 2}^{0}$ were investigated by Doppler-free laser spectroscopy techniques in 1986 and 1987 [2, 3]. The ns $(n=23,25-27,29-31)$ and $n d(n=22-33)$ Rydberg levels were observed by two-step laser excitation and detection techniques also in 1987 [4], the $n s(n=10-22)$ and nd $(n=8-20)$ series were determined by stepwise laser excitation in 1989 and 1990 [5, 6], and the 9s level was observed in 1996 [7]. Meanwhile, the ionization potential was determined in 1988 [8] and refined in 1989 [5]. More recently, an interferometric method was used to improve the accuracy of the 7S-7P transition frequency of francium by 1 order of magnitude in 2009 [9]. The theoretical investigation of the energy structure of francium could be outlined as follows. In 1995, many-body perturbation theory in screened Coulomb interaction was used to calculate energy levels, $E 1$ transition amplitudes, and the parity-nonconserving $E 1$ amplitude of the $7 \mathrm{~s}-8 \mathrm{~s}$ transition in francium by Dzuba et al. [10]. In 1998, Ritz formalism was utilized to predict the francium Rydberg $n s, n p$, and $n d$ levels up to $n=$ 30 [12]. In 1999, removal energies of the $n=7-10$ states and hyperfine constants of the $n=7$ and 8 states in francium were calculated by Safronova et al. [11], the calculations were based on the relativistic single-double approximation in which single and double excitations of Dirac-Hartree-Fock wave functions are included to all orders in perturbation theory. In 2004, pseudorelativistic HartreeFock method including core-polarization effects was used to investigate the radiative parameters for electric dipole transitions in the ions Ra II, Ac III, Th IV, and U VI along the francium isoelectronic sequence by Biémont et al. [13]. Also in 2004, relativistic Hartree-Fock method with several correlations was used to perform $a b$ initio calculation of isotope shifts for isotopes of francium (from $A=207$ to $A=228$ ) by Dzuba et al. [14]. In 2005, radiative corrections to $E 1$ matrix elements for $n s-n p$ transitions in the alkalimetal atoms lithium through francium are evaluated by Sapirstein and Cheng [15]. In 2006, relativistic third-order and all-order methods were used to calculate the scalar and tensor polarizabilities for the $5 f_{5 / 2}$ ground state in francium-like ion $\mathrm{Th}^{3+}$ by Safronova et al. [16]. In 2007, some $E 1$ transitions in the francium isoelectronic sequence were computed in the "Dirac-Fock + core-polarization" approximation by Migdalek and Glowacz-Proszkiewicz [17], in which the core-valence electron correlation was treated in a semiclassical picture. Also in 2007, relativistic manybody perturbation theory was applied to study energies of the $7 s, 7 p, 6 d$, and $5 f$ states of francium-like ions with nuclear charges $Z=87-100$, and also the transition rates, oscillator strengths, and lifetimes of francium-like ions with $Z=87-92$ 
TABLE 1: Spectral coefficients of the four energy series for francium atom by fitting the experimental values.

\begin{tabular}{lcccc}
\hline & $a_{0}$ & $a_{1}$ & $a_{2}$ & $a_{3}$ \\
\hline$n s^{2} \mathrm{~S}_{1 / 2}$ & 5.07085 & 0.323921 & 10.4546 & 96.28960 \\
$n d^{2} \mathrm{D}_{3 / 2}$ & 3.42366 & 0.101560 & -0.18468 & -1.68691 \\
$n d^{2} \mathrm{D}_{5 / 2}$ & 3.40262 & 0.133411 & -0.117487 & -0.89660 \\
\hline
\end{tabular}

by Safronova et al. [18]. Most of the energy levels for neutral Francium have been compiled by Sansonetti in 2007 [19]

In all these theoretical works; however, Rydberg energy levels for highly excited states $(n>31)$ of francium-like atoms have not been efficiently studied. The reason is that no matter what approximation method (including manybody perturbation theory, relativistic single-double approximation, pseudo-relativistic Hartree-Fock method, relativistic third-order and all-order methods, Dirac-Fock + corepolarization' approximation method, and so on ) is used, it is hard to investigate the highly excited states for such a complicated system that the configuration of the ground state is $1 s^{2} 2 s^{2} 2 p^{6} 3 s^{2} 3 p^{6} 3 d^{10} 4 s^{2} 4 p^{6} 4 d^{10} 4 f^{14} 5 s^{2} 5 p^{6} 5 d^{10} 6 s^{2} 6 p^{6} 7 s$. A possible approach to solve this problem is to utilize model potential method. In a previous work [20], we have successfully calculated the Rydberg energy levels and the quantum defects of $1 s^{2} 2 s^{2} 2 p n s^{3} P_{2,1,0}^{0}(n=3-50)$ and $1 s^{2} 2 s^{2} 2 p n d^{3} F_{3,2}^{0}$ $(n=3-50)$ spectrum series for Carbon I atom by the weakest bound electron potential model theory (WBEPMT) [21-23]. In this paper, the WBEPMT is utilized to investigate the Rydberg energy levels and the quantum defects of $n s^{2} \mathrm{~S}_{1 / 2}$ $(n=8-50), n d^{2} \mathrm{D}_{3 / 2}(n=6-50)$ and $n d^{2} \mathrm{D}_{5 / 2}(n=6-50)$ spectrum series for francium atom. The calculated results are in excellent agreement with the 74 known experimentally measured levels [19] (the absolute difference is less than $0.03 \mathrm{~cm}^{-1}$ ), and 58 energy levels for highly excited states are predicted, which might be significant for experiment guidance.

\section{Theory of WBEPM}

In the weakest bound electron potential model theory (WBEPMT) [21-23], the term energy of an atom system is expressed as

$$
T=T_{\text {limit }}+E,
$$

where $T_{\text {limit }}$ is the ionization limit corresponding to a given spectrum series, and $E$ is the energy of the weakest bound electron (WBE), which is determined by the radial equation (in atom unit)

$$
\frac{1}{2} \frac{d^{2} R}{d r^{2}}+\frac{1}{r} \frac{d R}{d r}+\left[E-V(r)-\frac{\ell(\ell+1)}{2 r^{2}}\right] R=0 .
$$

The potential that suffered by the WBE is modeled as [23]

$$
V(r)=-\frac{Z^{\prime}}{r}+\frac{k(k+1)+2 k \ell}{2 r^{2}},
$$

in which $Z^{\prime}$ stands for the number of effective nuclear charge, and $k$ an adjustable parameter and is not necessarily an integer. The first term of (3) represents the Coulomb potential and the second term represents the dipole potential that originated from polarization.

Inserting (3) into (2), the radial equation becomes

$$
\frac{1}{2} \frac{d^{2} R}{d r^{2}}+\frac{1}{r} \frac{d R}{d r}+\left[E+\frac{Z^{\prime}}{r}-\frac{\ell^{\prime}\left(\ell^{\prime}+1\right)}{2 r^{2}}\right] R=0,
$$

with

$$
\ell^{\prime}=\ell+k
$$

The solution to (4) is known as

$$
\begin{gathered}
R_{n \ell}(r)=A \exp \left(-\frac{Z^{\prime} r}{n^{\prime}}\right) r^{\ell^{\prime}} L_{n-\ell-1}^{2 \ell^{\prime}+1}\left(\frac{2 Z^{\prime} r}{n^{\prime}}\right), \\
E=-R\left(\frac{Z^{\prime}}{n^{\prime}}\right)^{2},
\end{gathered}
$$

where $n^{\prime}=n+k, A$ is the normalization constant, and $R$ the Rydberg constant. Let

$$
\frac{Z^{\prime}}{n^{\prime}}=\frac{Z_{\text {net }}}{n-\delta_{n}}
$$

where $Z_{\text {net }}$ is the net charge number of the atomic kernel and $Z_{\text {net }}=1$ for atoms, $\delta_{n}$ is the quantum defect, the term energy described by (1) is turned into

$$
T=T_{\text {limit }}+E=T_{\text {limit }}-R\left(\frac{Z_{\text {net }}}{n-\delta_{n}}\right)^{2} .
$$

According to Martin's formula about quantum defect [24], $\delta_{n}$ can be expressed as

$$
\delta_{n}=\sum_{i=0}^{3} \frac{a_{i}}{\left(n-\delta_{0}\right)^{2 i}},
$$

where $\delta_{0}$ is the quantum defect of the lowest energy level in a given series, $a_{i}(i=0,1,2,3)$ are parameters that can all be obtained through the least-square fitting of (8) with the first few experimental data in a given spectrum series.

\section{Results and Discussion}

By using the experimental data of the first four lowest energy levels of a given spectrum series, parameters $a_{i}$ are fitted by least-square method, the results are listed in Table 1.

The Rydberg energy levels of the spectrum series of $n s^{2} \mathrm{~S}_{1 / 2}(n=8-50), n d^{2} \mathrm{D}_{3 / 2}(n=6-50)$, and $n d^{2} \mathrm{D}_{5 / 2}(n=$ 6-50) for francium atom are calculated by (9). The calculated energy levels, quantum defects, and the differences between calculated and measured energy levels [19] are shown in Table 2 to Table 4, respectively. The unit of energy levels is expressed in $\mathrm{cm}^{-1}$.

From Tables 2, 3, and 4, it can be seen that the calculated results are in excellent agreement with the known experimental data since the absolute difference is less than $0.03 \mathrm{~cm}^{-1}$.

Therefore, by using the experimental data of the first four lowest energy levels for a given spectrum series, the Rydberg 
TABLE 2: Comparison of the calculated and measured values of $n s^{2} S_{1 / 2}(n=8-50)$ energy levels series for francium atoms.

\begin{tabular}{|c|c|c|c|c|c|c|c|c|c|}
\hline$n$ & $T_{\mathrm{cal}}$ & $T_{\exp }$ & Diff. & $\delta_{\mathrm{cal}}$ & $n$ & $T_{\mathrm{cal}}$ & $T_{\exp }$ & Diff. & $\delta_{\mathrm{cal}}$ \\
\hline 8 & 19739.98 & 19739.98 & 0.00 & 5.10670 & 30 & 32672.29 & 32672.29 & 0.00 & 5.07131 \\
\hline 9 & 25671.00 & 25671.00 & 0.00 & 5.08998 & 31 & 32685.64 & & & 5.07128 \\
\hline 10 & 28310.617 & 28310.617 & 0.00 & 5.08263 & 32 & 32697.54 & & & 5.07125 \\
\hline 11 & 29718.909 & 29718.909 & 0.000 & 5.07883 & 33 & 32708.19 & & & 5.07122 \\
\hline 12 & 30559.490 & 30559.504 & 0.014 & 5.07662 & 34 & 32717.74 & & & 5.07120 \\
\hline 13 & 31101.518 & 31101.539 & 0.022 & 5.07523 & 35 & 32726.36 & & & 5.07117 \\
\hline 14 & 31471.443 & 31471.465 & 0.024 & 5.07429 & 36 & 32734.16 & & & 5.07115 \\
\hline 15 & 31735.158 & 31735.182 & 0.021 & 5.07363 & 37 & 32741.23 & & & 5.07114 \\
\hline 16 & 31929.768 & 31929.789 & 0.014 & 5.07315 & 38 & 32747.67 & & & 5.07112 \\
\hline 17 & 32077.478 & 32077.492 & 0.016 & 5.07279 & 39 & 32753.55 & & & 5.07110 \\
\hline 18 & 32192.235 & 32192.251 & 0.018 & 5.07250 & 40 & 32758.93 & & & 5.07109 \\
\hline 19 & 32283.162 & 32283.180 & 0.016 & 5.07228 & 41 & 32763.86 & & & 5.07108 \\
\hline 20 & 32356.428 & 32356.444 & 0.011 & 5.07210 & 42 & 32768.40 & & & 5.07107 \\
\hline 21 & 32416.329 & 32416.340 & 0.008 & 5.07195 & 43 & 32772.59 & & & 5.07106 \\
\hline 22 & 32465.929 & 32465.937 & 0.010 & 5.07183 & 44 & 32776.46 & & & 5.07104 \\
\hline 23 & 32507.46 & 32507.47 & 0.00 & 5.07173 & 45 & 32780.04 & & & 5.07104 \\
\hline 24 & 32542.59 & 32542.59 & 0.00 & 5.07164 & 46 & 32783.36 & & & 5.07103 \\
\hline 25 & 32572.55 & 32572.56 & 0.01 & 5.07156 & 47 & 32786.45 & & & 5.07102 \\
\hline 26 & 32598.33 & 32598.34 & 0.01 & 5.07150 & 48 & 32789.33 & & & 5.07101 \\
\hline 27 & 32620.66 & 32620.67 & 0.01 & 5.07144 & 49 & 32792.01 & & & 5.07100 \\
\hline 28 & 32640.14 & 32640.14 & 0.00 & 5.07139 & 50 & 32794.51 & & & 5.07100 \\
\hline 29 & 32657.22 & 32657.22 & 0.00 & 5.07135 & & & & & \\
\hline
\end{tabular}

TABLE 3: Comparison of the calculated and measured values of $n d^{2} \mathrm{D}_{3 / 2}(n=6-50)$ energy levels series for francium atoms.

\begin{tabular}{|c|c|c|c|c|c|c|c|c|c|}
\hline$n$ & $T_{\mathrm{cal}}$ & $T_{\text {exp }}$ & Diff. & $\delta_{\mathrm{cal}}$ & $n$ & $T_{\mathrm{cal}}$ & $T_{\text {exp }}$ & Diff. & $\delta_{\text {cal }}$ \\
\hline 6 & 16229.87 & 16229.87 & 0.00 & 3.43035 & 29 & 32681.11 & 32681.11 & 0.00 & 3.42381 \\
\hline 7 & 24244.03 & 24244.03 & 0.00 & 3.42887 & 30 & 32693.50 & 32693.50 & 0.00 & 3.42379 \\
\hline 8 & 27600.657 & 27600.657 & 0.000 & 3.42731 & 31 & 32704.57 & & & 3.42378 \\
\hline 9 & 29316.497 & 29316.497 & 0.000 & 3.42630 & 32 & 32714.49 & & & 3.42378 \\
\hline 10 & 30309.964 & 30309.962 & 0.002 & 3.42564 & 33 & 32723.42 & & & 3.42377 \\
\hline 11 & 30936.330 & 30936.325 & 0.005 & 3.42519 & 34 & 32731.49 & & & 3.42376 \\
\hline 12 & 31356.512 & 31356.506 & 0.006 & 3.42488 & 35 & 32738.81 & & & 3.42375 \\
\hline 13 & 31652.007 & 31652.000 & 0.007 & 3.42465 & 36 & 32745.46 & & & 3.42375 \\
\hline 14 & 31867.687 & 31867.682 & 0.005 & 3.42448 & 37 & 32751.53 & & & 3.42374 \\
\hline 15 & 32029.910 & 32029.909 & 0.001 & 3.42435 & 38 & 32757.08 & & & 3.42374 \\
\hline 16 & 32154.989 & 32154.979 & 0.010 & 3.42425 & 39 & 32762.17 & & & 3.42373 \\
\hline 17 & 32253.455 & 32253.449 & 0.006 & 3.42417 & 40 & 32766.85 & & & 3.42373 \\
\hline 18 & 32332.356 & 32332.354 & 0.002 & 3.42410 & 41 & 32771.15 & & & 3.42373 \\
\hline 19 & 32396.553 & 32396.552 & 0.001 & 3.42405 & 42 & 32775.13 & & & 3.42372 \\
\hline 20 & 32449.484 & 32449.483 & 0.001 & 3.42400 & 43 & 32778.81 & & & 3.42372 \\
\hline 21 & 32493.64 & 32493.64 & 0.00 & 3.42397 & 44 & 32782.22 & & & 3.42372 \\
\hline 22 & 32530.86 & 32530.86 & 0.00 & 3.42394 & 45 & 32785.39 & & & 3.42371 \\
\hline 23 & 32562.52 & 32562.52 & 0.00 & 3.42391 & 46 & 32788.34 & & & 3.42371 \\
\hline 24 & 32589.68 & 32589.68 & 0.00 & 3.42388 & 47 & 32791.08 & & & 3.42371 \\
\hline 25 & 32613.15 & 32613.15 & 0.00 & 3.42386 & 48 & 32793.65 & & & 3.42371 \\
\hline 26 & 32633.57 & 32633.57 & 0.00 & 3.42385 & 49 & 32796.04 & & & 3.42370 \\
\hline 27 & 32651.44 & 32651.44 & 0.00 & 3.42383 & 50 & 32798.29 & & & 3.42370 \\
\hline 28 & 32667.18 & 32667.18 & 0.00 & 3.42382 & & & & & \\
\hline
\end{tabular}


TABLE 4: Comparison of the calculated and measured values of $n d^{2} \mathrm{D}_{5 / 2}(n=6-50)$ energy levels series for francium atoms.

\begin{tabular}{|c|c|c|c|c|c|c|c|c|c|}
\hline$n$ & $T_{\mathrm{cal}}$ & $T_{\exp }$ & Diff. & $\delta_{\mathrm{cal}}$ & $n$ & $T_{\mathrm{cal}}$ & $T_{\exp }$ & Diff. & $\delta_{\mathrm{cal}}$ \\
\hline 6 & 16429.64 & 16429.64 & 0.00 & 3.41476 & 29 & 32681.39 & 32681.39 & 0.00 & 3.4028 \\
\hline 7 & 24332.93 & 24332.93 & 0.00 & 3.41028 & 30 & 32693.75 & 32693.75 & 0.00 & 3.40280 \\
\hline 8 & 27645.373 & 27645.373 & 0.000 & 3.40771 & 31 & 32704.79 & & & 3.40279 \\
\hline 9 & 29341.817 & 29341.817 & 0.000 & 3.40621 & 32 & 32714.69 & & & 3.40278 \\
\hline 10 & 30325.611 & 30325.605 & 0.006 & 3.40528 & 33 & 32723.60 & & & 3.40277 \\
\hline 11 & 30946.651 & 30946.643 & 0.008 & 3.40467 & 34 & 32731.66 & & & 3.40276 \\
\hline 12 & 31363.667 & 31363.655 & 0.012 & 3.40425 & 35 & 32738.96 & & & 3.40275 \\
\hline 13 & 31657.168 & 31657.155 & 0.013 & 3.40394 & 36 & 32745.60 & & & 3.40274 \\
\hline 14 & 31871.529 & 31871.514 & 0.015 & 3.40371 & 37 & 32751.65 & & & 3.40273 \\
\hline 15 & 32032.847 & 32032.821 & 0.026 & 3.40354 & 38 & 32757.19 & & & 3.40273 \\
\hline 16 & 32157.283 & 32157.274 & 0.009 & 3.40340 & 39 & 32762.27 & & & 3.40272 \\
\hline 17 & 32255.281 & 32255.275 & 0.006 & 3.40330 & 40 & 32766.94 & & & 3.40272 \\
\hline 18 & 32333.834 & 32333.827 & 0.007 & 3.40321 & 41 & 32771.24 & & & 3.40271 \\
\hline 19 & 32397.765 & 32397.761 & 0.004 & 3.40314 & 42 & 32775.21 & & & 3.40271 \\
\hline 20 & 32450.490 & 32450.488 & 0.002 & 3.40308 & 43 & 32778.88 & & & 3.40270 \\
\hline 21 & 32494.48 & 32494.48 & 0.00 & 3.40303 & 44 & 32782.29 & & & 3.40270 \\
\hline 22 & 32531.57 & 32531.57 & 0.00 & 3.40299 & 45 & 32785.45 & & & 3.40269 \\
\hline 23 & 32563.13 & 32563.13 & 0.00 & 3.40295 & 46 & 32788.40 & & & 3.40269 \\
\hline 24 & 32590.20 & 32590.20 & 0.00 & 3.40292 & 47 & 32791.14 & & & 3.40269 \\
\hline 25 & 32613.60 & 32613.60 & 0.00 & 3.40289 & 48 & 32793.70 & & & 3.40268 \\
\hline 26 & 32633.97 & 32633.97 & 0.00 & 3.40287 & 49 & 32796.09 & & & 3.40268 \\
\hline 27 & 32651.80 & 32651.79 & 0.01 & 3.40285 & 50 & 32798.33 & & & 3.40268 \\
\hline 28 & 32667.49 & 32667.49 & 0.00 & 3.40283 & & & & & \\
\hline
\end{tabular}

energy levels and the quantum defects of $n s^{2} \mathrm{~S}_{1 / 2}(n=8-50)$, $n d^{2} \mathrm{D}_{3 / 2}(n=6-50)$, and $n d^{2} \mathrm{D}_{5 / 2}(n=6-50)$ spectrum series for francium atom have been calculated by virtue of the WBEPMT, the calculated results are in excellent agreement with the 74 known experimentally measured levels, one exception $\left(22 d^{2} \mathrm{D}_{5 / 2}\right)$ is noticed, and 58 energy levels for highly excited states are predicted, which might be significant for experiment guidance.

This implies that the WBEPMT is not only reliable to investigate the Rydberg energy levels of CI, but also dependable to probe the Rydberg energy levels of FrI. Compared with many-body perturbation theory, relativistic single-double approximation, pseudo-relativistic HartreeFock method, relativistic third-order and all-order methods, and Dirac-Fock + core-polarization' approximation method, the advantage of WBEPMT is that the program code, which can be easily written by the Mathematica language, is simpler and the running time is much shorter. The disadvantage of WBEPMT; however, is that the wave functions of the Rydberg states cannot be obtained, which might be settled by combining the WBEPMT with other theories.

\section{Acknowledgments}

This work was supported by the Scientific Research Foundation of the State Human Resource Ministry for Returned Chinese Scholars, China (Grant No. 2005LXAH06) and the Research Foundation of Education Bureau of Anhui Province, China (Grant Nos. KJ2008A145 and 2002HBL05).

\section{References}

[1] S. Liberman, J. Pinard, H. T. Duong et al., "First evidence of an optical transition in francium atoms," Comptes rendus hebdomadaires des seances de l'Acadmie des sciences B, vol. 286, pp. 253-258, 1978.

[2] J. Bauche, H. T. Duong, P. Juncar et al., "Accurate wavenumbers of the first resonance doublet of francium atoms and relativistic contribution to isotope shifts in the resonance doublets of francium and caesium atoms," Journal of Physics $B$, vol. 19, no. 18, pp. L593-L599, 1986.

[3] H. T. Duong, P. Juncar, S. Liberman et al., "First observation of the blue optical lines of francium," Europhysics Letters, vol. 3, pp. 175-182, 1987.

[4] S. V. Andreev, V. S. Letokhov, and V. I. Mishin, "Laser resonance photoionization spectroscopy of Rydberg levels in Fr," Physical Review Letters, vol. 59, no. 12, pp. 1274-1276, 1987.

[5] E. Arnold, W. Borchers, M. Carre et al., "High-resolution laser spectroscopy of Fr ns and nd Rydberg levels," Journal of Physics $B$, vol. 22, no. 14, pp. L391-L398, 1989.

[6] E. Arnold, W. Borchers, H. T. Duong et al., "Optical laser spectroscopy and hyperfine structure investigation of the 102S, 112S, 82D, and 92D excited levels in francium," Journal of Physics B, vol. 23, pp. 3511-3420, 1990.

[7] J. E. Simsarian, W. Shi, L. A. Orozco, G. D. Sprouse, and W. Z. Zhao, " $7 S_{1 / 2} \rightarrow 9 S_{1 / 2}$ two-photon spectroscopy of trapped francium," Optics Letters, vol. 21, no. 23, pp. 1939-1941, 1996.

[8] S. V. Andreev, V. I. Mishin, and V. S. Letokhov, "Rydberg levels and ionization potential of francium measured by laser-resonance ionization in a hot cavity," Journal of 
the Optical Society of America B, vol. 5, pp. 2190-2198, 1988.

[9] S. Sanguinetti, R. Calabrese, L. Corradi et al., "Accurate measurements of transition frequencies and isotope shifts of laser-trapped francium," Optics Letters, vol. 34, no. 7, pp. 893895, 2009.

[10] V. A. Dzuba, V. V. Flambaum, and O. P. Sushkov, "Calculation of energy levels, E1 transition amplitudes, and parity violation in francium," Physical Review A, vol. 51, no. 5, pp. 3454-3461, 1995.

[11] M. S. Safronova, W. R. Johnson, and A. Derevianko, "Relativistic many-body calculations of energy levels, hyperfine constants, electric-dipole matrix elements, and static polarizabilities for alkali-metal atoms," Physical Review A, vol. 60, no. 6, pp. 4476-4487, 1999.

[12] E. Biémont, P. Quinet, and V. Van Renterghem, "Theoretical investigation of neutral francium," Journal of Physics B, vol. 31, no. 24, pp. 5301-5314, 1998.

[13] E. Biémont, V. Fivet, and P. Quinet, "Relativistic Hartree-Fock and Dirac-Fock atomic structure calculations in Fr-like ions $\mathrm{Ra}^{+}, \mathrm{Ac}^{2+}, \mathrm{Th}^{3+}$ and $\mathrm{U}^{5+}$, Journal of Physics B, vol. 37, no. 20, pp. 4193-4204, 2004.

[14] V. A. Dzuba, W. R. Johnson, and M. S. Safronova, "Calculation of isotope shifts for cesium and francium," Physical Review A, vol. 72, no. 2, Article ID 022503, 9 pages, 2005.

[15] J. Sapirstein and K. T. Cheng, "Calculation of radiative corrections to E1 matrix elements in the neutral alkali metals," Physical Review A, vol. 71, no. 2, Article ID 022503, 2005.

[16] U. I. Safronova, W. R. Johnson, and M. S. Safronova, "Excitation energies, polarizabilities, multipole transition rates, and lifetimes in Th IV," Physical Review A, vol. 74, no. 4, Article ID 042511, 2006.

[17] J. Migdalek and A. Glowacz-Proszkiewicz, "'Dirac-Fock + core-polarization' calculations of E1 transitions in the francium isoelectronic sequence," Journal of Physics B, vol. 40, no. 21, pp. 4143-4154, 2007.

[18] U. I. Safronova, W. R. Johnson, and M. S. Safronova, "Excitation energies, polarizabilities, multipole transition rates, and lifetimes of ions along the francium isoelectronic sequence," Physical Review A, vol. 76, no. 4, Article ID 042504, 2007.

[19] J. E. Sansonetti, "Spectroscopic data for neutral francium (Fr I)," Journal of Physical and Chemical Reference Data, vol. 36, no. 2, pp. 497-507, 2007.

[20] Ma Kun, Huang Shi-zhong, Yu Jia-ming et al., "Calculation of Rydberg energy levels for C I atom," Journal of Atomic and Molecular Physics, vol. 26, pp. 39-44, 2009.

[21] N. W. Zheng and H. W. Xin, "Successive ionization potentials of $4 \mathrm{fn}$ electrons within 'WBEPM' theory," Journal of Physics B, vol. 24, pp. 1187-1191, 1991.

[22] N. Zheng, D. Ma, R. Yang, T. Zhou, T. Wang, and S. Han, "Efficient calculation of the energy levels of the carbon group," Journal of Chemical Physics, vol. 113, no. 5, pp. 1681-1687, 2000.

[23] N. W. Zheng, T. Wang, D. X. Ma, T. Zhou, and J. Fan, "Weakest bound electron potential model theory," International Journal of Quantum Chemistry, vol. 98, no. 3, pp. 281-290, 2004.

[24] W. C. Martin, "Series formulas for the spectrum of atomic sodium (Na I)," Journal of the Optical Society of America, vol. 70, pp. 784-788, 1980. 

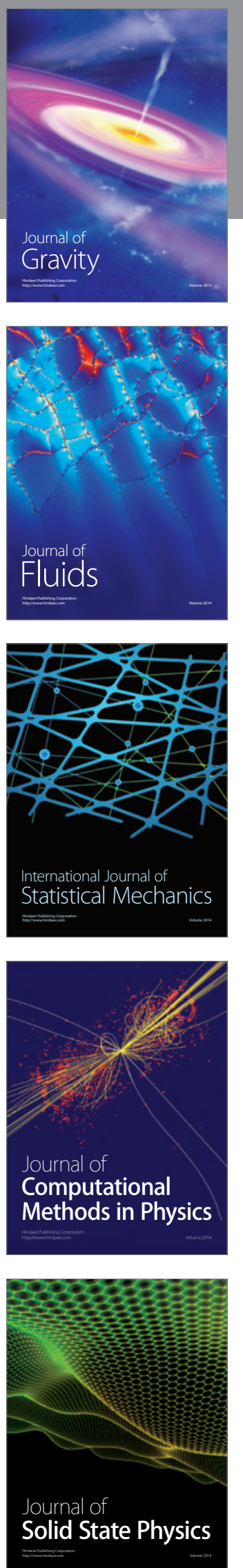

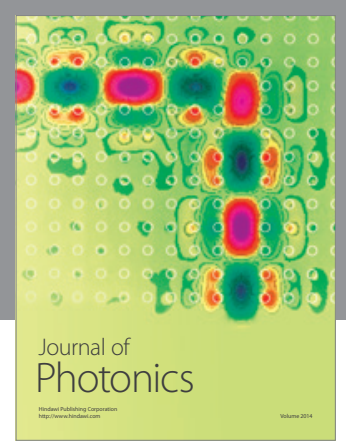

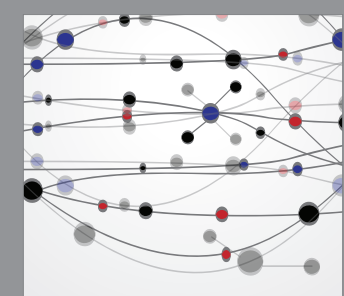

The Scientific World Journal
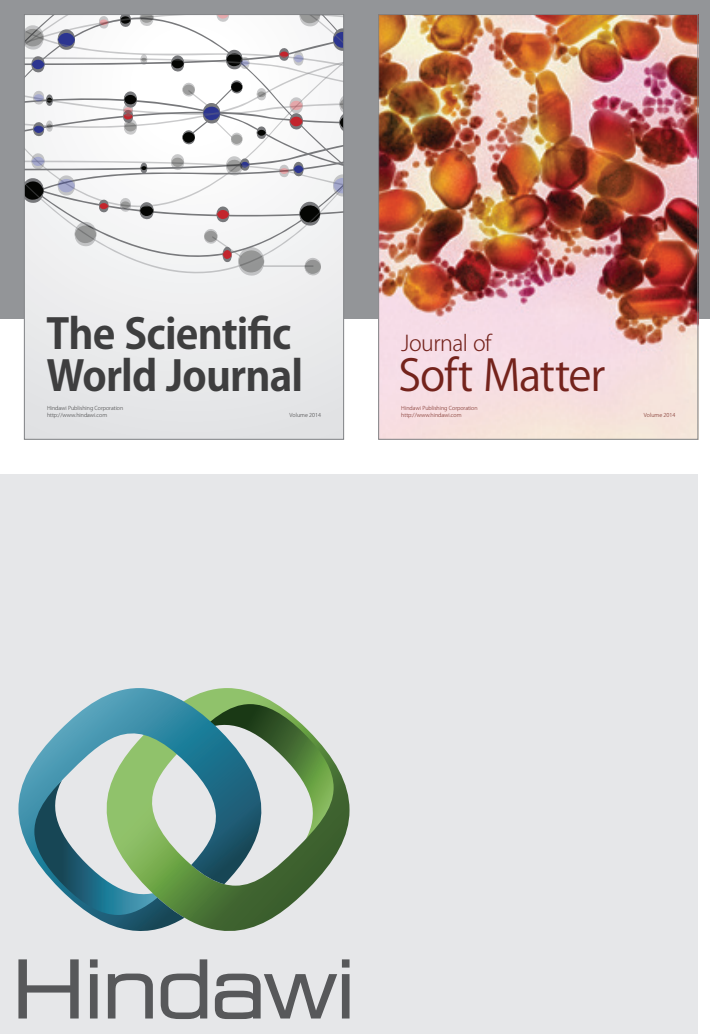

Submit your manuscripts at

http://www.hindawi.com
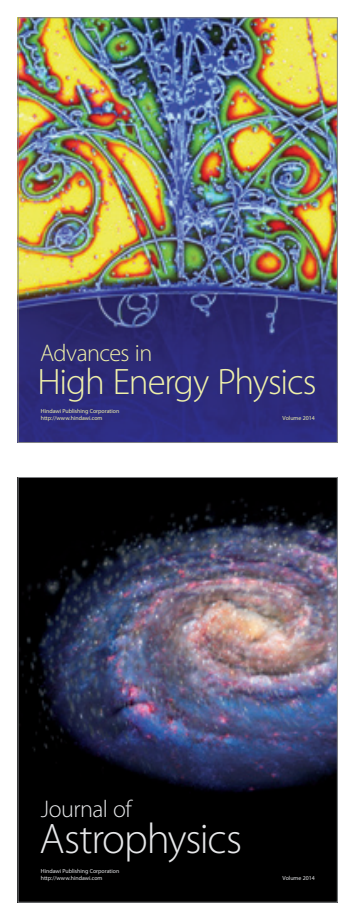
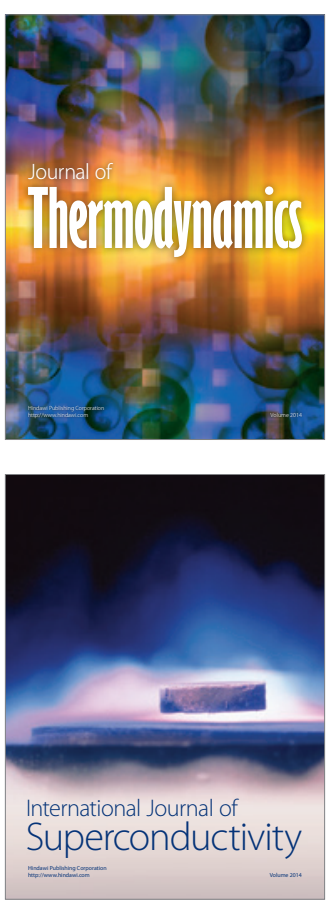
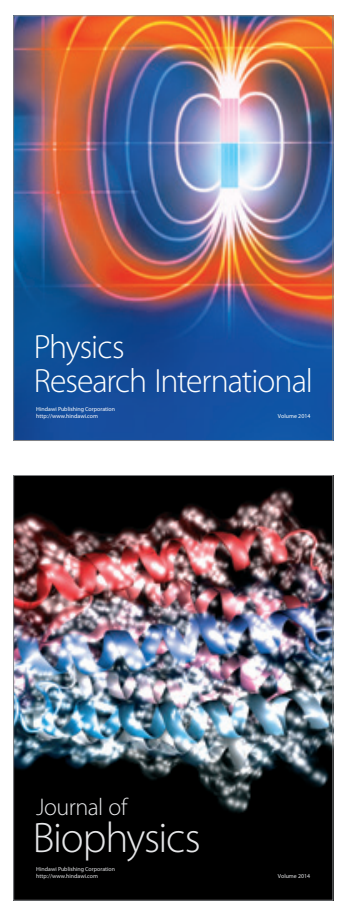
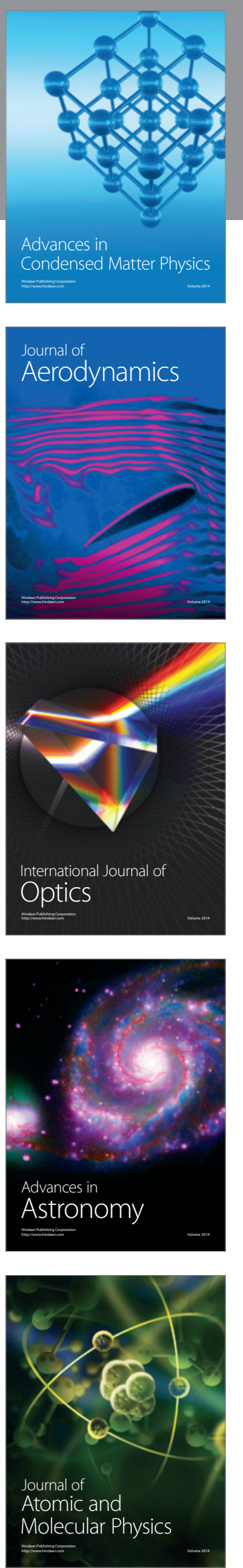